\title{
The Temperature Evolution of the Spectral Peak in High Temperature Superconductors
}

\author{
M. R. Norman ${ }^{1}$, A. Kaminski ${ }^{2}$, J. Mesot ${ }^{3}$, and J. C. Campuzano ${ }^{1,2}$ \\ (1) Materials Sciences Division, Argonne National Laboratory, Argonne, IL 60439 \\ (2) Department of Physics, University of Illinois at Chicago, Chicago, IL 60607 \\ (3) Laboratory for Neutron Scattering, ETH Zurich and PSI Villigen, CH-5232 Villigen PSI, Switzerland
}

\begin{abstract}
Recent photoemission data in the high temperature cuprate superconductor Bi2212 have been interpreted in terms of a sharp spectral peak with a temperature independent lifetime, whose weight strongly decreases upon heating. By a detailed analysis of the data, we are able to extract the temperature dependence of the electron self-energy, and demonstrate that this intepretation is misleading. Rather, the spectral peak loses its integrity above $T_{c}$ due to a large reduction in the electron lifetime.
\end{abstract}

74.25.Jb, 74.72.Hs, 79.60.Bm

Angle resolved photoemission spectroscopy (ARPES) has emerged as a powerful tool for understanding the electronic structure of high temperature cuprate superconductors. This has occurred because of its unique energy and momentum resolved nature, and its interpretation in terms of the single particle spectral functiond.

Perhaps the most novel behavior which has been observed is the dramatic rearrangement of the ARPES lineshape at the $(\pi, 0)$ points of the Brillouin zone when cooling below $T_{c}$. Above $T_{c}$, one has a single broad spectrum, indicating incoherent states. Below, though, a gap opens up in this incoherent spectrum, and inside this gap, a sharp peak emerges, leading to the well-known peak-diphump structurel which was first observed in tunneling spectral 3 . This onset of coherence at $T_{c}$ is quite dramatic, and has obvious implications for the microscopics of high temperature superconductorst.

How the sharp peak appears below $T_{c}$, though, has

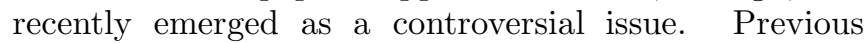
analysis 5 pointed to a dramatic increase in the low-energy lifetime as the source of its appearance. This agrees with interpretations of microwavel and thermald conductivity measurements Recently, though, this picture has been challengeds to. Based primarily on new high resolution data, it has been asserted that the spectral peak width in energy remains fixed with temperature. Rather, its weight (the quasiparticle renormalization factor, $z$ ) tracks the temperature dependence of the superconducting order parameter, and thus vanishes as $T_{c}$ is approached from below. As emphasized by Carlson et al. 11 , this is not in support of the previous "lifetime catastrophe" scenario, and casts doubt on standard interpretations based on reduction of the low energy scattering rate due to the opening of the superconducting gap 1214.

This new picture has a certain appeal to it, in that as Feng et al. show, one can draw a strong correlation between the temperature dependence of the spectral peak weight and other quantities, such as the superfluid density and the intensity of the magnefic resonance observed by inelastic neutron scattering 10 . Similar conclu- sions have been reached by Ding et al.15, though their analysis has some aspects of the lifetime catastrophe scenario as well. Moreover, since the spectrum is completely incoherent above $T_{c}$, it is natural to suppose that $z$ does indeed monotonically decrease to zero as $T_{c}$ is approached from below.

In this paper, we will argue that this new picture is misleading. Our analysis is based on a methodology we have developed where the electron self-energy is directly extracted from ARPES data16. From this analysis, we find that the quasiparticle residue $z$ is at best a marginally defined quantity at low temperatures, and becomes increasingly ill defined as $T$ increases. Moreover, any reasonable attempt to define a $z$ leads to a quantity which actually increases with increasing $T$ (more properly, the energy integrated spectral weight for binding energies less than the dip energy is temperature independent). This is due to a reduction in the mass renormalization as $T$ increases, similar to what is obtained from a generalized Drude analysis of optical data17. This, coupled with the increase of the low energy scattering rate (as also seen in optics), leads to a strong increase in the spectral peak width. That is, the spectral peak loses its integrity (rather than simply disappearing) due to a "lifetime catastrophe".

The ARPES data were taken on an optimally doped $\left(T_{c}=90 \mathrm{~K}\right)$ Bi2212 sample, with the $\Gamma-M$ axis parallel to the photon polarization vecter, and were previously reported in another connection 18 . The measurements were carried out at the Synchrotron Radiation Center in Madison, WI, on the U1 undulator beamline, with a Scienta SES 200 electron analyzer having an energy resolution of $16 \mathrm{meV}$ and a momentum resolution of $0.01 \AA^{-1}$.

In Fig. 1, we show data taken at the $M(\pi, 0)$ point of the Brillouin zone as a function of temperature. The leading edge of the spectral peak is determined by the superconducting gap, whose energy stays fairly fixed in temperature, and persists above $T_{c}$ (the pseudogap). On the trailing edge, one sees a spectral dip, whose energy also remains fixed in temperature, and becomes filled in 
above $T_{c}$. As these two energy scales define the two sides of the peak, this then gives the illusion that the peak width is independent of temperature. That such is not the case can be clearly seen by a closer inspection of the trailing edge of the peak. There is no question from Fig. 1 that the trailing edge is broadening with temperature, and this broadening is what is causing the spectral dip to fill in above $T_{c}$.

The earlier attempts to quantify this behavior involved separating the spectrum into a coherent ("peak") part and an incoherent ("hump") part. Although this a relatively straightforward procedure at low temperatures, where these two features are quite well defined, this becomes increasingly difficult as the temperature is raised and the spectral dip is filled in. The resulting ambiguity of what to call the coherent part, and what the incoherent part, has led to the differing conclusions in the literature.

The obvious way to overcome this difficulty is to treat the spectral function as a unified object. We note that the spectral function is defined as the imaginary part of the Greens function, that is

$$
A=-\frac{1}{\pi} \frac{\operatorname{Im} \Sigma}{(\omega-\epsilon-\operatorname{Re} \Sigma)^{2}+(\operatorname{Im} \Sigma)^{2}}
$$

where $\epsilon$ is the bare energy and $\Sigma$ the Dyson self-energy. Therefore, the temperature evolution of the total spectral function is determined by the temperature evolution of the self-energy.

We now briefly review the work of Ref. 16, where the procedure for extracting $\Sigma$ from ARPES data was developed. To determine $\Sigma$ uniquely, we need to know both $R e G$ and $I m G$. Unfortunately, from ARPES, we only know the occupied part of $\operatorname{Im} G$,

$$
I(\mathbf{k}, \omega)=C_{\mathbf{k}} \sum_{\delta \mathbf{k}} \int d \omega^{\prime} A\left(\mathbf{k}^{\prime}, \omega^{\prime}\right) f\left(\omega^{\prime}\right) R\left(\omega-\omega^{\prime}\right)+B
$$

where $I$ is the photocurrent, $C$ an intensity prefactor (proportional to the square of the dipole matrix element between initial and final states), $R$ the energy resolution function, and $B$ an extrinsic background, with the sum representing the momentum resolution. To make progress, certain assumptions have to be made. For instance, if we assume the spectrum is particle-hole symmetric, then the full $\operatorname{Im} G$ (symmetrized data) can be obtained from the above equation by the identity, $A(\omega) f(\omega)+A(-\omega) f(-\omega)=A(\omega)$, which holds even in the presence of resolution. Obviously, the particle-hole symmetry assumption is a reasonable approximation only on the Fermi surface. Since the spectral peak is dispersionless around $(\pi, 0)$, we will assume particle-hole symmetry for our purposes.

A more difficult problem is that the spectrum is a continous function of energy, and contains an extrinsic background, $B$. Separating out what part of the spectrum is intrinsic, and what is due to the state near the Fermi energy, and what is due to the tail of the main valence band, is an unresolved issue. For our purposes here, we will make the minimal assumption that the entire spectrum is intrinsic, which we simply cut off at the lowest energy data were taken at $\left(\omega_{c}=-320 \mathrm{meV}\right)$. As discussed extensively in Ref. 16, varying the cut-off and background assumptions make quantitative, rather than qualitative, differences in the results. This was verified in the present paper as well.

Therefore, our procedure is as follows. The photocurrent is symmetrized about zero energy (zero energy determined by measuring the chemical potential of a polycrystalline gold sample in electrical contact with the sample). The prefactor $C$ in Eq. 2 is eliminated by invoking the condition that the integral of $A$ is unity over the energy range considered $\left( \pm \omega_{c}\right)$, and $B$ is assumed zero. This then gives $\operatorname{Im} G$. ReG is determined by KramersKronig transformation. From the full $G, \operatorname{Re} \Sigma$ and $\operatorname{Im} \Sigma$ is then uniquely determined ( $\epsilon$ being taken as zero). Unlike Ref. 16, since the data were obtained from a high resolution detector, we elected to use raw data, i.e., the noise was not filtered out, nor the resolution deconvolved.

In Fig. 2a, $\operatorname{Im} \Sigma$, as determined from Fig. 1, is plotted for various temperatures. At low temperatures and energies, it is characterized by a peak centered at zero energy due to the superconducting energy gap, and a "normal" part which can be treated as a constant plus an $\omega^{2}$ term. Besides the constant term, which is surprisingly large, this is the expected form for the self-energy for a superfluid Fermi liquid. A maximum in $\operatorname{Im} \Sigma$ occurs near the energy of the spectral dip. Beyond this, $\operatorname{Im} \Sigma$ has a large, nearly frequency independent, value (the slow decay at higher energies is due to the cut-off at $-320 \mathrm{meV}$, and so should not be taken seriously). As the temperature is raised, the zero energy peak broadens, the constant term increases, and the $\omega^{2}$ term goes away.

In Fig. 2b, the quantity $\omega-R e \Sigma$ is plotted. The low temperature, low energy behavior is again characteristic of a superfluid Fermi liquid. At low energies, there is a $1 / \omega$ term due to the energy gap, and a "normal" part which is linear in $\omega$. As expected, the zero crossing is near the location of the spectral peak. Beyond this, there is a minimum near the specral dip energy, then the data are approximately linear again, but with a smaller slope than near the zero crossing. As the temperature is raised, the gap $(1 / \omega)$ term broadens out and the low energy linear in $\omega$ term decreases, paralleling the behavior discussed above for $\operatorname{Im} \Sigma$.

To further quantify this behavior, we have found that the following form for the self-energy gives a good description of data for low energies (smaller than the energy of the spectral dip)

$$
\begin{gathered}
\omega-\operatorname{Re} \Sigma=Z \omega\left(1-\frac{\Delta_{r}^{2}}{\omega^{2}+\Gamma_{r}^{2}}\right) \\
-\operatorname{Im} \Sigma=c+c_{F L} \omega^{2}+\frac{\Delta_{i}^{2} \Gamma_{i}}{\omega^{2}+\Gamma_{i}^{2}}
\end{gathered}
$$

A discussion of this form is in order. The term involving 
$\Delta$ is simply a broadened version of the BCS self-energy, with $\Gamma$ representing a combination of resolution and pair lifetime effects]. Note that the quantity $\Delta_{i} / \sqrt{Z}$ in the expression for $\operatorname{Im} \Sigma$ is approximately equivalent to $\Delta_{r}$ where $Z$ is the mass factor in the expression for $\operatorname{Re} \Sigma$, and that $\Gamma_{r}$ and $\Gamma_{i}$ are essentially equivalent (these quantities would be identical by the Kramers-Kronig relations if the self-energy had this form for all energies). From this expression, one sees that the occupied quasiparticle weight is approximately (neglecting $\Gamma$ ) the inverse of $2 Z$ (the other half of the quasiparticle weight lies above the Fermi energy). Also, if the gap term is neglected, one sees that the effective quasiparticle width is approximately $-\operatorname{Im} \Sigma / Z$. Note that $Z$ is the inverse of the quasiparticle renormalization factor, $z$. The above assumes, of course, that quasiparticles exist, a matter which we will address below.

In Fig. 3, the temperature variation of these coefficients, obtained from fitting data over an energy range of $\pm 60 \mathrm{meV}$, is shown. The most significant finding is that $Z$ decreases with temperature. This implies that if quasiparticles exist, their weight increases with perature. This is in contrast with earlier analyses 90 , 1.5 . We have attempted to quantify the weight in two ways. First, we define $z$ by taking the inverse of the derivative of $\omega-\operatorname{Re} \Sigma$ from Eq. 3 evaluated at the peak maximum, $\omega_{p}$, and multiplying by two (the factor of two accounting for the other peak above the Fermi energy). Second, we input Eq. 3 into Eq. 1, defining a quantity $A_{\text {low }}$, integrate this in energy over the full energy range $( \pm 320$ $\mathrm{meV}$ ) and divide by the actual integrated weight. These quantities ( $z$ and ratio) are plotted in Fig. 4a, and both increase with increasing $T$. This conclusion is further supported by the fact that the actual integrated weight over the energy range of the fit $( \pm 60 \mathrm{meV})$ relative to the total integrated weight is essentially constant with temperature (quantity low of Fig. 4a). For this to be true, then the weight factor must increase to compensate for the broadening of the peak.

Therefore, rather than the peak decreasing in weight, it disappears by broadening strongly in energy. This can be seen directly by inspecting Fig. 2, in that as the temperature increases, $\operatorname{Im} \Sigma$ (Fig. 2a) in the vicinity of the peak increases in magnitude with $T$, and $Z$ (roughly the slope in Fig. 2b near the zero crossing) decreases with $T$. In fact, it is the strong variation of $c$ and $Z$, and the fact that they operate in concert, which is responsible for the rapid variation in the effective width of the peak with $T$. This can be quantified by two estimates of the width of the peak which are analogous to the weight estimates discussed above, first from the quantity $-z \operatorname{Im} \Sigma\left(\omega_{p}\right)$, second from the full width half maximum (FWHM) of $A_{\text {low }}$. These quantities $(F W 1$ and $F W 2)$ are plotted in Fig. $4 \mathrm{~b}$. Note, the FWHM ( $F W 2$ ) would be even larger above $T_{c}$ if it were not for the pseudogap splitting the peak in two.

The above analysis is important in that it shows how coherence is lost in the system. It is apparent from Figs. 1 and 2 that once a temperature is reached where the peak is no longer discernable in the data, the difference in behavior of the self-energy between low energies and high energies is lost. That is, once the spectral dip is filled in, the low and high energy behaviors have merged, and the sharp peak and broad hump at low temperature is simply replaced by a single broad peak (with a leading edge gap due to the pseudogap). In fact, as a cautionary remark, it is obvious from Fig. 4b, where the energy of the spectral peak is compared to the peak width, that if anything, quasiparticles are at best marginally defined below $80 \mathrm{~K}$, and become ill defined above this. This is exactly the temperature at which the weights plotted in Fig. 4a start to increase. Still, everything is consistent with the spectral peak simply losing its integrity as the temperature is raised. Certainly, the analysis here in no way supports a picture of a well defined quasiparticle peak whose weight simply disappears upon heating. An analysis was also done with a background subtraction, and similar conclusions were found.

Our results have significant implications for microscopic theories of the cuprates. In previous work $19,13,20$, we have argued that the spectral lineshape at $(\pi, 0)$ is naturally explained by the coupling of the electrons to a magnetic resonance seen by neutron scattering. Since the intensity of this resonance decreases with temperature, then the coupling of the electrons to this mode also decreases, and thus one would expect $Z$ to decrease, just as we find in Fig. 3a. As the neutron resonance intensity decreases, the spin gap in the dynamic susceptibility fills in, which we speculate is responsible for the "filling in" of $\operatorname{Im} \Sigma$ seen in Fig. 2a. The combination of these two effects causes the spectral peak to rapidly broaden with temperature, losing its integrity above $T_{c}$.

In conclusion, we have studied the thermal evolution of the electron self-energy by direct analysis of ARPES data, and come to the conclusion that the spectral peak below $T_{c}$ broadens out above $T_{c}$ due to a "lifetime catastrophe", rather than the quasiparticle weight $z$ monotonically decreasing to zero.

We thank Mohit Randeria for discussions. This work was supported by the U. S. Dept. of Energy, Basic Energy Sciences, under contract W-31-109-ENG-38, and the National Science Foundation DMR 9974401.

${ }^{1}$ M. Randeria, et al., Phys. Rev. Lett. 74, 4951 (1995).

${ }^{2}$ D. S. Dessau, et al., Phys. Rev. Lett. 66, 2160 (1991).

${ }^{3}$ Q. Huang, et al., Phys. Rev. B 40, 9366 (1989).

${ }^{4}$ A. J. Millis, Nature 398, 193 (1999).

${ }^{5}$ M. R. Norman, M. Randeria, H. Ding, and J. C. Campuzano, Phys. Rev. B 57, R11093 (1998).

${ }^{6}$ D. A. Bonn, P. Dosanjh, R. Liang, and W. N. Hardy, Phys. Rev. Lett. 68, 2390 (1992).

${ }^{7}$ K. Krishana, J. M. Harris, and N. P. Ong, Phys. Rev. Lett. 
75, 3529 (1995).

${ }^{8}$ A. G. Loeser, et al., Phys. Rev. B 56, 14185 (1997).

${ }^{9}$ A. V. Federov, et al., Phys. Rev. Lett. 82, 2179 (1999).

${ }^{10}$ D. L. Feng, et al., Science 289, 277 (2000).

${ }^{11}$ E. W. Carlson, D. Orgad, S. A. Kivelson, and V. J. Emery, Phys. Rev. B 62, 3422 (2000).

${ }^{12}$ Y. Kuroda and C. M. Varma, Phys. Rev. B 42, 8619 (1990).

13 M. R. Norman and H. Ding, Phys. Rev. B 57, R11089 (1998).

14 A. Abanov and A. V. Chubukov, Phys. Rev. Lett. 83, 1652 (1999).

${ }^{15}$ H. Ding, et al., preprint cond-mat/0006143.

${ }^{16}$ M. R. Norman, et al., Phys. Rev. B 60, 7585 (1999).

${ }^{17}$ A. V. Puchkov, D. N. Basov, and T. Timusk, J. Phys. Cond. Matter 8, 10049 (1996).

18 A. Kaminski, et al., preprint cond-mat/0004482.

19 M. R. Norman, et al., Phys. Rev. Lett. 79, 3506 (1997).

${ }^{20}$ J. C. Campuzano, et al., Phys. Rev. Lett. 83, 3709 (1999).

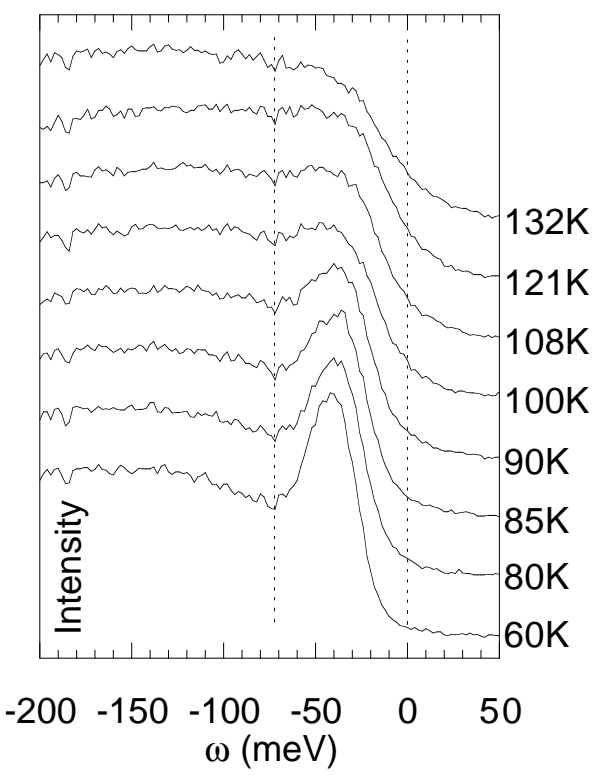

FIG. 1. Temperature dependence of ARPES data at $(\pi, 0)$ for a $T_{c}=90 \mathrm{~K}$ Bi2212 sample. The vertical dotted lines mark the spectral dip energy and the chemical potential.

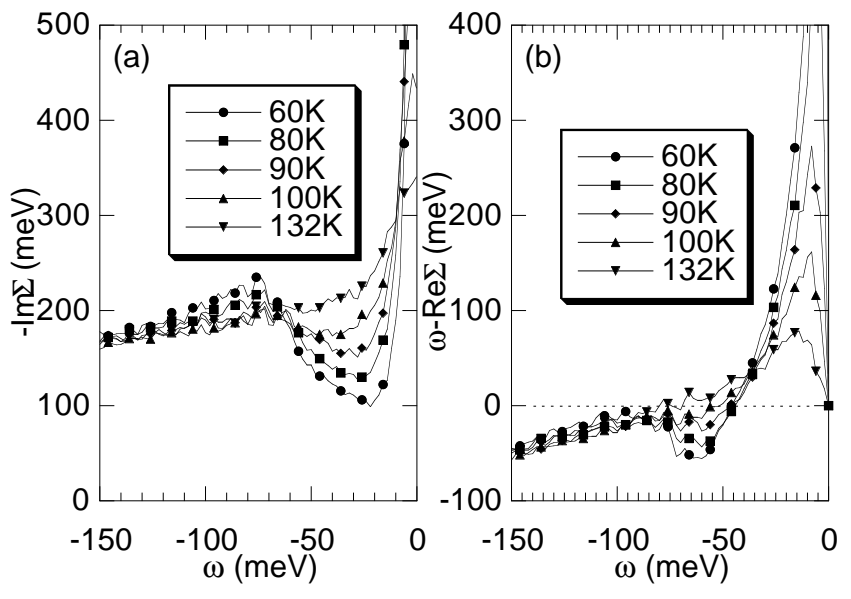

FIG. 2. Temperature dependence of (a) the imaginary and (b) real parts of the self-energy derived from the data of Fig. 1.
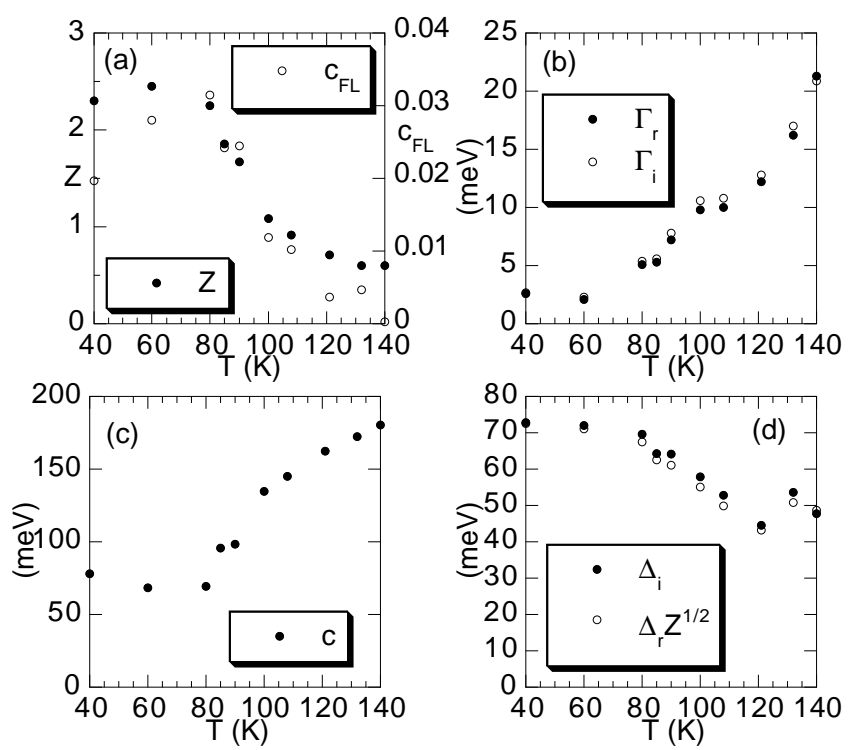

FIG. 3. Temperature dependence of the various coefficients of the self-energy from Eq. 3 obtained from fits of Fig. 2 over an energy range of $\pm 60 \mathrm{meV}$.
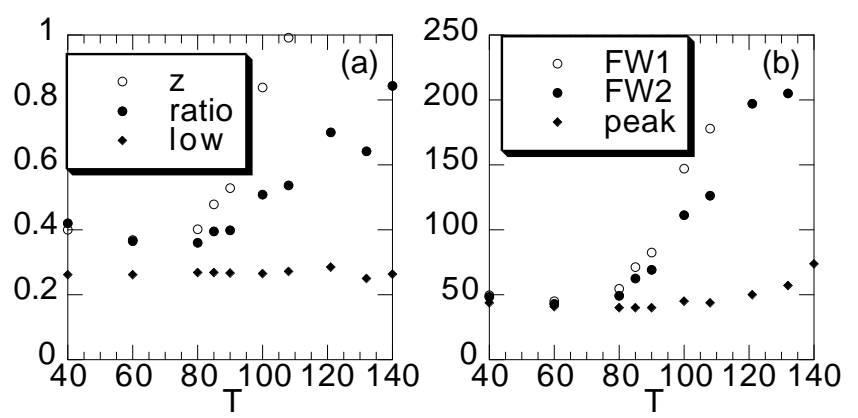
FIG. 4. Temperature dependence of (a) the spectral peak weight ( $z$ and ratio) and (b) the width (FWHM) of the spectral peak ( $F W 1$ and $F W 2$ ) as defined in the text. Also plotted is the low energy weight (low) and the peak energy (peak). For $F W 2$, the low energy edge of the peak is either defined by the HWHM, or zero energy if $A$ at zero energy is greater than half the maximum. 